\title{
Evaluation of Several Screening Approaches for Detection of Cervical Lesions in Rural Shandong, China
}

\author{
Li-Ju Zong ${ }^{1}$, You-Zhong Zhang ${ }^{1 *}$, Xing-sheng Yang ${ }^{1}$, Jie Jiang ${ }^{1}$, Bao-Xia Cui ${ }^{1}$, \\ Yun-Bo Qiao ${ }^{1}$, Li Li ${ }^{1}$, Kan Jiang ${ }^{1}$, Wen-Jing Zhang ${ }^{1}$, Bei-Hua Kong ${ }^{1}$, Keng Shen ${ }^{2}$
}

\begin{abstract}
Purpose: The study was designed to: (1) investigate the prevalence of high-risk human papillomavirus (HRHPV) infection and cervical neoplasia; and (2) evaluate clinical performance of visual inspection with acetic acid/Lugol's iodine (VIA/VILI), Pap smear, high-risk human papillomavirus (HR-HPV) DNA test for detecting cervical intraepithelial neoplasia grade 2 or worse (CIN2+) and (3) explore appropriate screening approach in rural areas of Shandong Province. Materials and Methods: A total of 3,763 eligible women from Yiyuan County in Yimeng mountainous areas of rural Shandong, China, were enrolled and underwent Pap smear, HR-HPV DNA testing by Hybrid Capture 2 (HC2), and VIA /VILI tests. Women positive in any test were referred to colposcopy and biopsy as indicated. Results: The prevalence of HR-HPV infection among all enrolled women was $11.1 \%$ and that in healthy women was $9.9 \%$. In total 33 cases of CIN1, 16 cases of CIN2, 6 cases of CIN3 but none of cervical cancer were detected and the crude prevalence of CIN2+ was $0.58 \%$. For detecting CIN2+, the sensitivity of HR-HPV DNA testing, VIA/VILI, Pap smear was $90.9 \%, 77.3 \%, 81.8 \%$, respectively. Pap smear had the best specificity of $98.2 \%$, followed by HR-HPV DNA testing with specificity of 89.4\%, VIA/VILI had the lowest specificity of $81.2 \%$. Colposcopy referral rate of HR-HPV DNA testing, VIA/VILI, Pap smear was $11.1 \%, 18.5 \%, 2.3 \%$, respectively. Conclusions: Our results suggest that HR-HPV DNA testing alone might be appropriate for primary cervical cancer screening in rural low-resource areas of Shandong Province, China.
\end{abstract}

Keywords: Cervical cancer screening - high-risk human papillomavirus DNA - rural areas - Shandong, China

Asian Pac J Cancer Prev, 16 (5), 1907-1912

\section{Introduction}

Cervical cancer is one of the most common cancers among women globally, with most of them (more than 80\%) occurring in developing countries (Arbyn et al., 2011; Jemal et al., 2011). The People's Republic of China, as a developing country, contains approximately one fifth of the world's population and has about $64 \%$ of population resident in rural areas. The disease burden in rural China is still heavy due to lack of infrastructure and resources necessary for routine Papanicolaou (Pap) smear screening, histological diagnosis, and proper treatment procedures (Shi et al., 2012a). Conventional Pap smear, though reduced cervical cancer incidence and prevalence rates widespread, has its limitations: requiring highly trained personnel, adequately equipped laboratories, and referral systems to communicate results (Massad et al., 2013). Visual inspection with acetic acid (VIA) and visual inspection with Lugol's iodine (VILI) are two modifications of a direct visual assessment of the cervix. Visual inspection with acetic acid/ Lugol's iodine (VIA /VILI) could be used as primary screening in low-resource settings for their advantages: requiring a lower level of infrastructure, less expensive, immediate result (Sankaranarayanan et al., 2012). High-risk human papillomavirus (HR-HPV) types deoxyribonucleic acid (DNA) test has been taken as an alternative primary screening test considering the fact that cervical cancers and a high proportion of cervical precursors are caused by persistent HR-HPV infection (Schiffman et al., 2011). The Digene Hybrid Capture 2 (HC2) for HR-HPV DNA testing (QIAGEN, Gaithersburg, MD), approved by the Food and Drug Administration, can offer an objective assay for presence of the viruses. Pap smear, VIA, VILI, HR-HPV test have their characteristics and indications in different settings (Longatto et al., 2012a).

While these screening approaches have been evaluated in several studies (Sarian et al., 2005; Gravitt et al., 2010; Longatto et al., 2012b), few studies have reported about the prevalence of HR-HPV infection and cervical neoplasia in rural Shandong Province with different socioeconomic factors. Thus we addressed the following aims among 
Li-Ju Zong et al

women aged 30 65 years in a population-based study in Yiyuan County, located in Yimeng mountainous areas of rural Shandong, China: (1) to investigate the prevalence of HR-HPV infection and cervical neoplasia, (2) to evaluate the performance of VIA/VILI, Pap smear, and HR-HPV DNA testing for detecting cervical intraepithelial neoplasia grade 2 or worse $(\mathrm{CIN} 2+)$, and explore an appropriate screening approach in rural areas of Shandong Province, (3) to provide free cervical cancer screening service for women in rural Yiyuan County.

\section{Materials and Methods}

\section{Ethical considerations}

The study protocol was approved by the Institutional Review Boards at Qilu Hospital of Shandong University. Written informed consent was obtained by signature or thumbprint.

\section{Recruitment and data collection}

From June to October in 2010 year, totally 3763 women were enrolled in this project. Women aged 3065 years were considered eligible if they were married or had previous sexual intercourse; had a cervix; had not been previously diagnosed with cervical cancer; were not pregnant; were physically able to undergo routine cervical cancer screening; were able to provide informed consent. Before all clinical study procedures, eligible women were given a presentation including an explanation of informed consent, the aim of this project, risk and benefits of participation, knowledge about HPV infection and cervical cancer prevention, encouragement for participation. Following the presentation, women who agreed to participate provided a signature or thumbprint on the printed consent form. After consent, women responded to a brief questionnaire designed to collect information on demographics, socioeconomic factors, reproductive history and contact information. These women also underwent a gynecological examination, including a conventional Pap smear and collection of cervical cells in sample transport medium for HR-HPV DNA testing by HC2. Thereafter, VIA/VILI test were performed.

\section{Pap smear and interpretation}

After removing any obscuring mucus from the cervix with a cotton swab, cervical cells were collected using an Ayre's spatula and endocervical brush, smeared onto a glass slide and fixed in ethanol. Slides were stained according to standard protocols, and reviewed by a local cytopathologist and external cytopathologist (senior experts from Qilu Hospital). Pap smears results were categorized according to the 2001 Bethesda System (Solomon et al., 2002). For analysis, the results equal or worse than atypical squamous cells of undetermined significance (ASCUS) were considered positive.

\section{HR-HPV DNA testing and interpretation}

The HR-HPV DNA testing was done by $\mathrm{HC} 2$, using cervical cell swabs, as previously described in detail (Kjaer et al., 2008). Briefly, cervical cell swabs were collected using HC2 kit brushes, and placed into $1 \mathrm{ml}$ of
Digene standard transport medium. Samples were stored at $-20^{\circ} \mathrm{C}$ and sent to Qilu Hospital for analysis. The samples were analyzed only for the presence of $13 \mathrm{HR}-\mathrm{HPV}$ types namely $16,18,31,33,35,39,45,51,52,56,58,59$, and 68 . The results were classified positive if relative light unit coefficient (RLU/CO) was equal to or greater than 1.0 RLU/CO.

\section{VIA /VILI methods and interpretation}

After sample collection for Pap smear and HC2, 5\% acetic acid was applied on the cervix through embedded cotton. The cervix was examined by naked eyes with a bright lamp after 1 minute. Thereafter use of acetic acid, Lugol's iodine was also applied on the cervix through embedded cotton. Immediately after, the cervix was observed in the same manner. Results of VIA and VILI were classified as normal, abnormal, and suggesting cancer according to the examiner's visual impression. Examiners have been trained to classify their visual impression according to the Atlas of Visual Inspection. Abnormal or suggesting cancer in VIA or VILI was classified positive.

\section{Colposcopy and biopsy}

Women who had at least one positive screening test were referred to colposcopy. All of examinations were performed by experienced and certified colposcopists who were aware that referred patients had at least one positive screening test, but were masked to the specific test results. Any suspicious lesions were subjected to directed biopsy for histological confirmation. Endocervical curettage (ECC) was performed for diagnosis if the entire squamocolumnar junction (SCJ) could not be visualized or a lesion extended into the endocervical canal. All biopsies were read by local pathologists, and reviewed by an expert pathologist from Qilu Hospital who was blinded to the original diagnosis. Histological diagnosis were categorized following the classification system as normal, inflammation/cervicitis, squamous metaplasia, cervical intraepithelial neoplasia Grade1, 2, or 3 (CIN1, CIN2, or CIN3), microinvasive cancer, squamous cervical cancer (SCC), adenocarcinoma in situ(AIS), and cervical adenocarcinoma. Histology results were considered gold standard diagnosis and true disease status. Women with the results CIN2+ were treated by Loop Electrosurgical Excision Procedure (LEEP) or Cold Knife Conization (CKC) or hysterectomy.

\section{Data analysis}

Women were considered to have a normal cervix or negative result if: $i$ ) all screening tests were negative; $i i)$ any screening test was positive but colposcopy revealed no lesion; or iii) any screening test and colposcopy was abnormal, but histological results were normal/ inflammation. Women were considered as cases of cervical diseases if histological results were CIN2 or worse. Crude estimates of diagnostic accuracy were biased due to non-random exclusion of women who did not have the opportunity for full diagnostic verification (Mahé and Gaffikin, 2005). Diagnostic accuracy statistics including sensitivity, specificity, and positive/negative predictive 
values (PPV/NPV) and 95\% confidence interval (CI) were calculated for detecting CIN2+ with each Pap smear, VIA/ VILI and HPV testing. As previously described (Denny et al., 2000), we calculated crude sensitivity, that is the ratio of the number of test positive, disease positive women to the total number of women identified with disease. This measure is roughly equivalent to sensitivity, but does not require that the biopsy be applied to all women examined or verification bias be corrected. We calculated the crude specificity in the same way as previously reported (Denny et al., 2000), that is the ratio of the number of test negative, disease negative women to the total number of women free of disease. Pearson's chi-square $\left(\chi^{2}\right)$ test or Fisher's exact test or Yates' continuity correction chi-square test were used to assess differences in prevalence of HPV infection by age and disease grade and differences in sensitivity and specificity for CIN2+ results. Multiple test comparisons were compared using Bonferroni adjusted $\chi^{2}$ test. The diagnosis agreement between local cytopathologist and experts from Qilu Hospital was measured using kappa statistics. All analyses were conducted using the SPSS for Windows Version 16.0 statistical package (SPSS Inc., Chicago, IL).

\section{Results}

\section{Demographic characteristics}

A total of 3763 eligible women were enrolled in the study (Table 1). The median age was 40 years with 1438 $(38.2 \%)$ in the age group 40-49 years. A total of 2317 (61.6\%) women had one or two childbirths, 1392 women $(37.0 \%)$ reported three or more births. Only 78 (2.1\%) women were illiterate, most of women $(95.1 \%)$ had received primary or middle school education.

\section{Overview of screening and diagnostic procedures}

As is shown in Figure 1, among 3763 women, 417 (11.1\%,95\%CI: 10.1-12.1) women were detected positive in HR-HPV DNA testing, 698 (18.5\%, 95\%CI: 17.3-19.8) women were defined as positive in VIA/VILI test, 87 $(2.3 \%, 95 \% \mathrm{CI}$ : $1.8-2.8)$ women were detected positive in Pap smear (i.e. equal or worse than ASCUS). Of the 3763 Pap smears that were taken, 3734 (99\%) were classified as being "satisfactory for evaluation". We considered

Table 1. Demographic Characteristics of the Women

\begin{tabular}{lcc}
\hline Characteristics & Number & Percentage \\
\hline Participants & 3763 & \\
Age (years) & & \\
$\quad 30-39$ & 1824 & 48.5 \\
$\quad 40-49$ & 1438 & 38.2 \\
$50-59$ & 439 & 11.7 \\
$\quad \geq 60$ & 62 & 1.6 \\
Total number of childbirth & & \\
$\quad 0$ & 54 & 1.4 \\
$1-2$ & 2317 & 61.6 \\
$\quad \geq 3$ & 1392 & 37.0 \\
Education & & \\
$\quad$ Illiterate & 78 & 2.1 \\
$\quad$ Primary & 1673 & 44.5 \\
$\quad$ Middle & 1904 & 50.6 \\
$\quad$ High & 108 & 2.8 \\
\hline
\end{tabular}

the few unsatisfactory Pap smear results as Pap negative rather than repeating Pap smear, which will not be feasible logistically in rural areas. Total $1031(27.4 \%)$ women with positive results in any test were referred to colposcopy, of these, 654 women had indication for biopsy and considered as negative for CIN2+. In the referred group, $377(10.0 \%)$ women underwent biopsy and pathological diagnosis. We detected a total of 33 CIN1 cases, 16 CIN2 cases , $6 \mathrm{CIN} 3$ cases and none of cervical cancer.

\section{Prevalence HPV infection and cervical disease}

Among 3763 women with HR-HPV DNA testing by $\mathrm{HC} 2$ results, the prevalence of was $11.1 \%$ (Table2). Though there was no statistical difference of HR-HPV

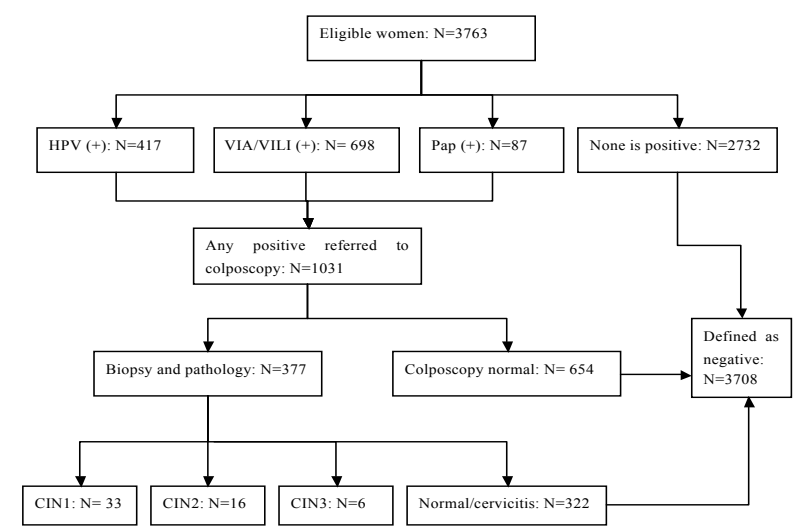

Figure 1. Overview of the Screening and Diagnostic Procedures

Table 2. Distribution of HPV Infection in Ages and Disease Grades

\begin{tabular}{lrcc}
\hline Variables & Number & $\begin{array}{c}\text { HPV } \\
\text { positive } \\
\text { cases }\end{array}$ & $\begin{array}{c}\text { HPV } \\
\text { infection } \\
\text { rate }(\%)\end{array}$ \\
\hline Total & 3763 & 417 & 11.1 \\
\hline Age (years) & 1824 & 205 & 11.2 \\
30-39 & 1438 & 168 & 11.7 \\
40-49 & 439 & 40 & 9.1 \\
50-59 & 62 & 4 & 6.5 \\
60-65 & & & \\
Cervix & 3708 & 370 & 9.9 \\
$\quad$ Normal/cervicitis & 33 & 27 & 81.8 \\
CIN1 & 16 & 14 & 87.5 \\
CIN2 & 6 & 6 & 100 \\
CIN3 & & & \\
\hline
\end{tabular}

Table 3. Test Positive Cases by 3 Tests in Different Cervical Grades

\begin{tabular}{lccc}
\hline & \multicolumn{3}{c}{ Screening tests } \\
\cline { 2 - 4 } Einal diagnosis & HR-HPV DNA test & VIA/VILI & Pap smear \\
Negative(N=3708) & 370 & 658 & 50 \\
CIN1(N=33) & 27 & 23 & 19 \\
CIN2(N=16) & 14 & 12 & 12 \\
CIN3(N=6) & 6 & 5 & 6 \\
$<$ CIN2(N=3741) & 397 & 681 & 69 \\
CIN2+(N=22) & 20 & 17 & 18 \\
Totalnumber & 417 & 698 & 87 \\
\hline
\end{tabular}

*HPV DNA: human papillomavirus DNA; CIN: cervical intraepithelial neoplasia; CIN2+: CIN2 or worse; VIA: visual inspection with acetic acid; VILI: visual inspection with Lugol's iodine 
Table 4. Clinical performance of the 3 Screening Tests for Detection of CIN2+

\begin{tabular}{lccrrr}
\hline Screen tests & \multicolumn{3}{c}{ Clinical performance of tests } \\
\cline { 2 - 6 } & Sensitivity(95\%CI) & Specificity(95\%CI) & PPV & NPV & $\begin{array}{r}\text { Colposcopy } \\
\text { referral rate }\end{array}$ \\
\hline HR-HPV DNA & $90.9(78.9-99.9)$ & $89.4(88.4-90.4)$ & 47.9 & 99.9 & 11.1 \\
VIA/VILI & $77.3(59.8-94.8)$ & $81.2(80.6-83.0)$ & 24.4 & 99.8 & 18.5 \\
Pap smear & $81.8(65.7-97.9)$ & $98.2(97.7-98.6)$ & 20.7 & 99.8 & 2.3 \\
\hline
\end{tabular}

*PPV: positive predictive value; NPV: negative predictive value; CI: confidence interval; CIN: cervical intraepithelial neoplasia; CIN2+: CIN2 or worse; HPV DNA: human papillomavirus DNA; VIA: visual inspection with acetic acid; VILI: visual inspection with Lugol's iodine

Table 5. Pap Smears Results of Local and External Cytopathologist

\begin{tabular}{lccr}
\hline Final diagnosis & \multicolumn{2}{c}{ Local diagnosis } & In total \\
\cline { 2 - 3 } & Positive & Negative & \\
\hline Positive & 83 & 4 & 87 \\
Negative & 40 & 3636 & 3676 \\
In total & 123 & 3640 & 3763 \\
\hline
\end{tabular}

*Kappa $=0.78$; Local diagnosis were determined by local cytopathologist, final diagnosis were dependent on expert cytopathologist from Qilu Hospital. The results equal or worse than atypical squamous cells of undetermined significance (ASCUS) were considered positive

DNA prevalence between age categories, the HR-HPV infection rate in age from 60 to 65 years was the lowest. HR-HPV prevalence in CIN groups, ranging from $81.8 \%$ to $100 \%$, was significantly different from that in normal/ cervicitis with $9.9 \%$ positive rate. But the differences between CIN grades were not significant statistically. The crude prevalence of CIN2, CIN3, CIN2+ was $0.42 \%$, $0.16 \%, 0.58 \%$, respectively, without considering the 'true positive' cases in the defined negative group that could be detected if the entire enrolled population had underwent colposcopy and biopsy.

\section{Clinical performance of the three screening tests}

Test positive cases by 3 tests are reported in Table 3 . Of the defined negative group ( $\mathrm{N}=3708)$, the most cases (658) were detected positive in VIA/VILI test, that were false positive cases, while only 50 positive cases were detected in Pap smear test. Among the CIN2+ $(\mathrm{N}=22$, including 14 CIN2 and 6 CIN3) group, the positive cases (i.e. true positive case) in HC2, VIA/VILI, Pap smear test was $20,17,18$, respectively.

The clinical performance characteristics of the 3 screening tests are reported in Table 4 . The sensitivity of HC2 HPV DNA testing, VIA/VILI, Pap smear for detecting CIN2+ was $90.9 \%, 77.3 \%, 81.8 \%$ respectively. There was no significant difference between tests statistically $(p>0.1)$. Pap smear had the best specificity of 98.2\%, followed by HR-HPV DNA testing with specificity of $89.4 \%$, VIA/VILI had the lowest specificity of $81.2 \%$. The specificities were different significantly in statistics $(p<0.001)$. As for colposcopy referral rate, Pap smear had the lowest referral rate of $2.3 \%$, VIA/VILI possessed the highest referral rate of $18.5 \%$ compared with moderate $11.1 \%$ referral rate of HR-HPV DNA test $(p<0.05)$. HRHPV DNA test had the highest PPV of $47.9 \%$ and Pap smear had the lowest PPV of $20.7 \%$, while VIA/VILI had the moderate PPV of $24.4 \%$. These tests had NPV of above $99 \%$, the differences of NNP in these 3 tests were not significant.

\section{Evaluation of cytological results by local pathologist}

The agreement between the local and external (Qilu Hospital) cytopathologist was evaluated by kappa statistic (Table 5): the agreement was $98.8 \%$ and $x=0.78$. The final diagnosis was determined by cytopathologist from Qilu Hospital. The results equal or worse than atypical squamous cells of undetermined significance (ASCUS) were considered positive. Of 123 positive results by local diagnosis, only 83 cases were confirmed as positive, 40 cases were overdiagnosed. Of 3640 negative results by local cytopathologist, 4 cases were diagnosed as positive.

\section{Discussion}

The present research was designed to provide cervical cancer screening service and evaluate the clinical performance of several screening approaches for the detection of cervical neoplasia in rural areas of Shandong Province, China. It was conducted in Yiyuan County of Yimeng mountainous areas, a typical county of many resource-poor settings in Shandong.

To the present study, we found that the prevalence of HR-HPV infection in this region among all enrolled women was $11.1 \%$ and that in healthy women was $9.9 \%$. The prevalence of HR-HPV infections in healthy women was similar to that $(9.61 \%)$ reported in littoral region of Shandong Province (Yuan et al., 2011), also similar to $9.2 \%$ of global average (Clifford et al., 2005), but somewhat lower than that $(15-22 \%$ range) observed in studies of other provinces in China (Dai et al., 2006; Wu et al., 2007; Sun et al., 2010; Wang et al., 2014). We detected total 16 cases of CIN2 and 6 cases of CIN 3 but none of cervical cancer. The prevalence of cervical precancerous disease (CIN2+), i.e. high-grade squamous intraepithelial lesion (HSIL) was $0.58 \%$, obviously lower than $3.7 \%$, the estimate prevalence of HSIL in women aged 30-54 years in a pooled summary (Shi et al., 2012a). We noted that most of women $(63 \%)$ had two or less childbirths. Considering the fact that parity number is an important factor on development of cervical cancer, we assumed the lower prevalence of CIN2+ was related to the strict onechild family policy in Shandong Province (Huang, 1982; Hesketh et al., 2005). The uncorrected verification bias may also account for this lower prevalence of CIN2+. On the other hand, the low number of cases detected in our study perhaps reflects an underdiagnosis of disease. The case definition was based on histological confirmation of CIN2+ by colposcopically-directed biopsy (CDB). This is a flawed reference standard and likely to miss a substantial proportion of CIN2+ lesions (Jeronimo and 
Schiffman, 2006).

VIA screening is the simplest method of screening with the cost estimate for $\$ 2.64$ per test in China and greatest relative ease of use (Shi et al., 2012b). The approach does not require high technology and has been demonstrated to reduce the deaths of women in developing countries (Wright and Kuhn, 2012). In our study, the sensitivity of VIA/VILI was 77.3\% (95\%CI: 59.8-94.8), similar to previous report (Chen et al., 2012). However, several weaknesses of VIA and VILI have been revealed, particularly the high rate of false-positive cases, which may lead to substantial number of colposcopy (Pimple and Shastri, 2014). The colposcopy referral rate of VIA/ VILI in this study was $18.5 \%$, leading to loss of followup and resource waste. It has been reported that a strong decline in the sensitivity of the VIA test in women aged 40 years or older ( $\mathrm{Li}$ et al., 2009), showing the relatively poor performance.

Pap smear or cytology is the most common screening method in developed areas and decreased the incidence of advanced cervical cancer and associated mortality (Mukakalisa et al., 2014). However, Pap smears are challenging to perform in developing countries because the process requires high-quality laboratories, qualified lab technicians and trained cytopathologists, which may not be available in developing countries (Bradley et al., 2006). In our study, Pap smear had the highest specificity of $98.2 \%$ and moderate sensitivity of $81.8 \%$. Another research reported similar corrected sensitivity of $80.2 \%$ for detecting HSIL (Moy et al., 2010). The overall agreement between the local and external (Qilu Hospital) cytopathologists was satisfactory. Notably, however, among the positive results of local diagnosis, almost half of them were false positive. The results of Pap smear are subjective and affected by variation in the technical skills of the gynecologists and pathologists. Scholars had identified VIA, and HPV DNA test as recommended alternatives to Pap smears in developing countries (Maine et al., 2011).

The HR-HPV DNA testing by HC2 detected the most CIN2+ cases and had the moderate specificity of $89.4 \%$ and colposcopy referral rate of $11.1 \%$. For women in rural areas, they may just have once or twice cervical cancer screening in their lifetime. So a strategy with high sensitivity might be preferable at the cost of specificity loss. However, low specificity could result in the overreferral of women and limit overall screening coverage (Moy et al., 2010). HPV DNA testing has some limitations, including expensive cost requiring a laboratory, and at least 7 hours needed to process. The alternative to HRHPV DNA testing in low-resource-setting countries is careHPV, which is promising as a primary screening method for cervical cancer prevention in low-resource regions (Qiao et al., 2008), resulting in a reduction of morbidity and mortality of cervical cancer. Though PPV and NPV are affected by the prevalence of a disease, they can provide a different perspective for the performance of screening test in a specific population. HR-HPV DNA testing had the highest PPV for CIN2+ (47.9\%), followed by Pap smear (20.7\%), VIA/VILI (24.4\%).

The differences in the performance of the screening test in different populations as reported may be attributed by variation in the technical skills of the personnel, as well as differences in demographic characteristics of the populations screened. Considering the overall performance of 3 screening tests, our results indicate that HR-HPV DNA testing by $\mathrm{HC} 2$ alone might be appropriate for low-resource areas and countries. These findings align with the conclusion by Alliance for Cervical Cancer Prevention (ACCP) that HPV-DNA should become the standard test in developing countries, as well as a systematic review (Nahvijou et al., 2014). For HPVpositive women, cytology triage may be considered in settings with adequate infrastructure, whereas VIA triage may be suitable in settings with limited infrastructure (Muwonge et al., 2014).

We acknowledge that our study has limitations with respect to verification bias; women who resulted negative in all tests were not referred to colposcopy and did not receive random biopsy. Therefore, our results may overestimate the clinical sensitivity of the tests and underestimate the prevalence of cervical lesions. However, among the women with any positive results, we highly encouraged gynecologist to take a biopsy even if only a slight abnormality was observed during colposcopy. Furthermore, HR-HPV DNA testing was performed on all women together with VIA/VILI and Pap test; it is very likely that most women with CIN2+ were detected.

Our study has several strengths. Firstly, we provided free cervical cancer screening for women in rural Yiyuan County. Secondly, propagation and education about cervical cancer prevention contributed to compliance among women, unlike the report that non-compliance was a barrier for screening (Gravitt et al., 2010). Finally, this project was a population-based study and three screening tests were performed on each woman to compare directly clinical performance of screening approaches for detection of cervical neoplasia, which was not possible in large community randomized trials.

In conclusion, our results suggest that HR-HPV DNA testing alone might be appropriate for cervical cancer screening in rural low-resource areas of Shandong. It is important to emphasize performance of the screening tests, but the screening test is only one component of the population-based program required to reduce cervical cancer incidence and mortality. Effort to extend cervical cancer prevention strategies, HPV vaccine inoculation, precancerous lesion screening and treatment to Chinese women should be made, making cervical cancer be the first cancer eliminated (Qiao, 2010).

\section{Acknowledgements}

This research is supported by the Supporting Program of the "Eleventh Five-year Plan" for Science and Technology Research of China from Ministry of Health of the People's Republic of China (2008BAI57B01)

\section{References}

Arbyn M, Castellsague X, De Sanjose S, et al (2011). Worldwide burden of cervical cancer in 2008. Ann Oncol, 22, 2675-86. 
Bradley J, Coffey P, Arrossi S, et al (2006). Women's perspectives on cervical screening and treatment in developing countries: experiences with new technologies and service delivery strategies. Women health, 43, 103-21.

Chen C, Yang Z, Li Z, et al (2012). Accuracy of several cervical screening strategies for early detection of cervical cancer: a meta-analysis. Int J Gynecol Cancer, 22, 908-21.

Clifford GM, Gallus S, Herrero R, et al (2005). Worldwide distribution of human papillomavirus types in cytologically normal women in the International Agency for Research on Cancer HPV prevalence surveys: a pooled analysis. Lancet, 366, 991-8.

Dai M, Bao YP, Li N, et al (2006). Human papillomavirus infection in shanxi province, people's republic of China: a population-based study. Br J Cancer, 95, 96-101.

Denny L, Kuhn L, Pollack A, et al (2000). Evaluation of alternative methods of cervical cancer screening for resource-poor settings. Cancer, 89, 826-33.

Gravitt PE, Paul P, Katki HA, et al (2010). Effectiveness of VIA, Pap, and HPV DNA testing in a cervical cancer screening program in a peri-urban community in Andhra Pradesh, India. PLoS One, 5, 13711.

Hesketh T, Lu L, Xing ZW (2005). The effect of China's onechild family policy after 25 years. $N$ Engl J Med, 353, 1171-6.

Huang LJ (1982). Planned fertility of one-couple/one-child policy in the People's Republic of China.J Marriage Family, 44, 775-84.

Jemal A, Bray F, Center MM, et al (2011). Global cancer statistics. CA Cancer J Clin, 61, 69-90.

Jeronimo J, Schiffman M (2006). Colposcopy at a crossroads. Am J Obstet Gynecol, 195, 349-53.

Kjaer SK, Breugelmans G, Munk C, et al (2008). Populationbased prevalence, type-and age-specific distribution of $\mathrm{HPV}$ in women before introduction of an HPV-vaccination program in Denmark. Int J Cancer, 123, 1864-70.

Li N, Shi JF, Franceschi S, et al (2009). Different cervical cancer screening approaches in a Chinese multicentre study. $\mathrm{Br} J$ Cancer, 100, 532-7.

Longatto-Filho A, Naud P, Derchain SF, et al (2012a). Performance characteristics of Pap test, VIA, VILI, HRHPV testing, cervicography, and colposcopy in diagnosis of significant cervical pathology. Virchows Archiv, 460, 577-85.

Longatto-Filho A, Naud P, Derchain SF, et al (2012b). Performance characteristics of Pap test, VIA, VILI, HRHPV testing, cervicography, and colposcopy in diagnosis of significant cervical pathology. Virchows Arch, 460, 577-85.

Mahe C, Gaffikin L (2005). Screening test accuracy studies: how valid are our conclusions? application to visual inspection methods for cervical screening. Cancer Causes Control, 16, 657-66.

Maine D, Hurlburt S, Greeson D (2011). Cervical cancer prevention in the $21^{\text {st }}$ century: cost is not the only issue. Am J Public Health, 101, 1549-55.

Massad LS, Einstein MH, Huh WK, et al (2013). 2012 updated consensus guidelines for the management of abnormal cervical cancer screening tests and cancer precursors. Obstetrics Gynecol, 121, 829-46.

Moy LM, Zhao FH, Li LY, et al (2010). Human papillomavirus testing and cervical cytology in primary screening for cervical cancer among women in rural China: comparison of sensitivity, specificity, and frequency of referral. Int $J$ Cancer, 127, 646-56.

Mukakalisa I, Bindler R, Allen C, et al (2014). Cervical cancer in developing countries: effective screening and preventive strategies with an application in Rwanda. Health Care Women Int, 35, 1065-80.

Muwonge R, Wesley RS, Nene BM, et al (2014). Evaluation of cytology and visual triage of human papillomavirus-positive women in cervical cancer prevention in India. Int J Cancer, 134, 2902-9.

Nahvijou A, Hadji M, Marnani AB, et al (2014). A systematic review of economic aspects of cervical cancer screening strategies worldwide: discrepancy between economic analysis and policymaking. Asian Pac J Cancer Prev, 15, 8229-37.

Pimple S, Shastri S (2014). Comparative evaluation of human papilloma virus-DNA test verses colposcopy as secondary cervical cancer screening test to triage screen positive women on primary screening by visual inspection with $5 \%$ Acetic acid. Indian J Cancer, 51, 117.

Qiao Y-1, Sellors JW, Eder PS, et al (2008). A new HPV-DNA test for cervical-cancer screening in developing regions: a cross-sectional study of clinical accuracy in rural China. Lancet Oncol, 9, 929-36.

Qiao Y (2010). Perspective of cervical cancer prevention and control in developing countries and areas. Chin J Cancer, 29, 1-3.

Sankaranarayanan R, Nessa A, Esmy PO, et al (2012). Visual inspection methods for cervical cancer prevention. Best practice Res Clinical Obstetrics Gynaecol, 26, 221-32.

Sarian L, Derchain S, Naud P, et al (2005). Evaluation of visual inspection with acetic acid (VIA), Lugol's iodine (VILI), cervical cytology and HPV testing as cervical screening tools in Latin America: this report refers to partial results from the LAMS (Latin AMerican Screening) study. J Med Screen, 12, 142-9.

Schiffman M, Wentzensen N, Wacholder S, et al (2011). Human papillomavirus testing in the prevention of cervical cancer. $J$ Natl Cancer I, 103, 368-83.

Shi JF, Canfell K, Lew JB, et al (2012a). The burden of cervical cancer in China: synthesis of the evidence. Int J Cancer, 130, 641-52.

Shi JF, Chen JF, Canfell K, et al (2012b). Estimation of the costs of cervical cancer screening, diagnosis and treatment in rural Shanxi Province, China: a micro-costing study. BMC Health Serv Res, 12, 123.

Solomon D, Davey D, Kurman R, et al (2002). The 2001 Bethesda System: terminology for reporting results of cervical cytology. Jama, 287, 2114-9.

Sun ZR, Ji YH, Zhou WQ, et al (2010). Characteristics of HPV prevalence among women in Liaoning province, China. Int J Gynaecol Obstet, 109, 105-9.

Wang XC, Sun LQ, Ma L, et al (2014). Prevalence and genotype distribution of human papillomavirus among women from Henan, China. Asian Pac J Cancer Prev, 15, 7333-6.

Wright TC, Jr., Kuhn L (2012). Alternative approaches to cervical cancer screening for developing countries. Best Pract Res Clin Obstet Gynaecol, 26, 197-208.

Wu RF, Dai M, Qiao YL, et al (2007). Human papillomavirus infection in women in Shenzhen City, people's republic of China, a population typical of recent Chinese urbanisation. Int J Cancer, 121, 1306-11.

Yuan X, Yang Y, Gu D, et al (2011). Prevalence of human papillomavirus infection among women with and without normal cervical histology in Shandong Province, China. Arch Gynecol Obstet, 283, 1385-9. 